\title{
Integrated spectrometer and integrated detectors on Silicon-on-Insulator for short-wave infrared applications
}

\author{
E. Ryckeboer ${ }^{1,2}$, A. Gassenq ${ }^{1,2}$, N. Hattasan ${ }^{1,2}$, B. Kuyken ${ }^{1,2}$, L. Cerutti ${ }^{3}$, J.B. Rodriguez ${ }^{3}$, E. Tourniée ${ }^{3}$, \\ G. Roelkens ${ }^{1,2}$, W. Bogaerts ${ }^{1,2}$, R. Baets ${ }^{1,2}$ \\ ${ }^{1}$ Photonics Research Group, Department of Information Technology, Ghent University-imec, Ghent B-9000, Belgium \\ ${ }^{2}$ Center for Nano- and Biophotonics (NB-Photonics), Ghent University, Ghent B-9000, Belgium \\ ${ }^{3}$ University of Montpellier 2 - CNRS, UMR 5214, Montpellier 34095, France \\ Email: Eva.Ryckeboer@intec.ugent.be, Tel.: +32-9-264-8933
}

\begin{abstract}
We present a miniature spectrometer fabricated on a Silicon-on-Insulator substrate with center wavelength at $2.15 \mu \mathrm{m}$. The spectrometer is a planar concave grating (PCG) with 6 channels and $4 \mathrm{~nm}$ channel spacing with a crosstalk of $-12 \mathrm{~dB}$. We investigate heterogeneously integrated grating-assisted GaInAsSb photodiodes for future implementation as detector array.

OCIS codes: (130.3120) Integrated optics devices; (300.6190) Spectrometers
\end{abstract}

\section{Introduction}

Integrated spectrometers fabricated on the silicon-on-insulator (SOI) platform have been shown to be an attractive low-cost solution for the analysis of organic samples [1]. The target wavelengths of these spectrometers are mostly confined to the telecommunication wavelength range (1.3-1.65 $\mu \mathrm{m})$. However, by extending this range to 2-2.5 $\mu \mathrm{m}$ we can detect a larger variety of molecules and additionally, exploit stronger absorption bands. The SOI platform imposes no direct limits hereto as the waveguides are transparent for these longer wavelengths. Furthermore, straightforward scaling of the waveguide dimensions enables us to fabricate demultiplexers for 2-2.5 $\mu \mathrm{m}$ with good performance. Apart from passive routing components, photodetectors are required for a fully integrated spectrometer. In this work we use a quaternary GaInAsSb epitaxial layer stack, grown by molecular beam epitaxy, as the active photodetector material. This epitaxy is perfectly adapted for detection in the 2-2.5 $\mu \mathrm{m}$ range, given its bandgap of $2.65 \mu \mathrm{m}$ at room temperature. The $\mathrm{p}-\mathrm{i}-\mathrm{n}$ photodetectors are heterogeneously integrated on the waveguide circuit by employing benzocyclobutene (DVS-BCB) as an adhesive bonding layer. While in a previous realization the light from the waveguide was evanescently coupled to the photodetector [2], the light is now coupled vertically to the photodetectors using grating couplers. As a result, we can increase the thickness of the BCB-bonding layer, which makes the fabrication procedure more robust.

\section{Wavelength demultiplexer}
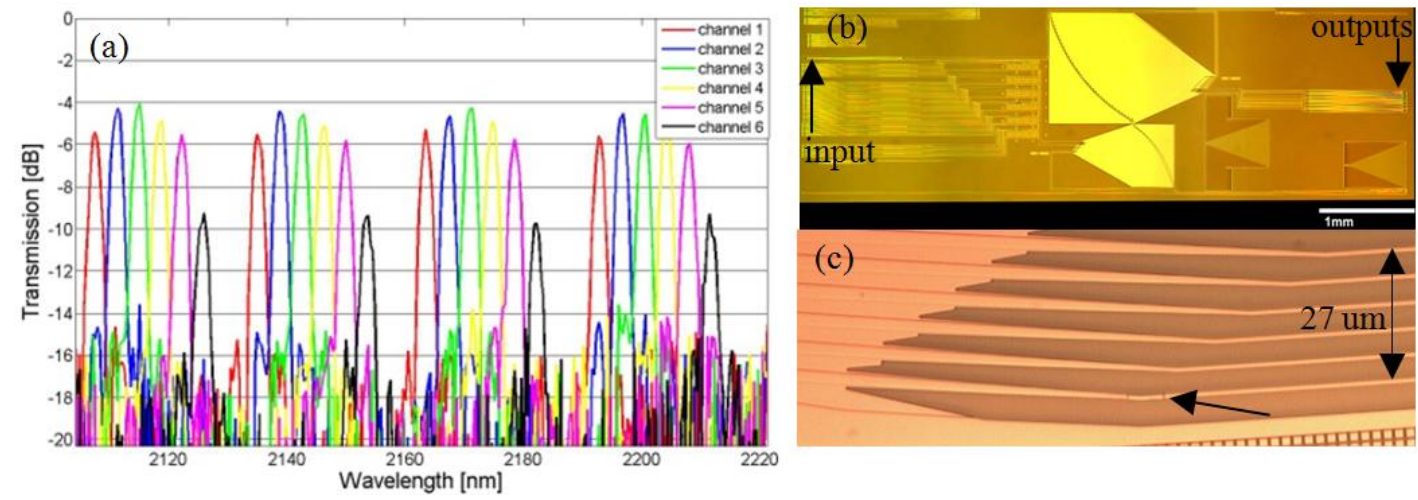

Fig. 1. (a) Transmission spectrum of 6-channel PCG for TE-polarization. (b) microscope picture of fabricated device (c) microscope picture of exit aperture of the $6^{\text {th }}$ channel

The demultiplexer is a planar concave grating (PCG) with 6 channels and $4 \mathrm{~nm}$ channel spacing, designed for TE polarization. It is fabricated in a CMOS pilot line on a $200 \mathrm{~mm}$ SOI wafer, comprising a 220-nm-thick Si waveguide layer on a $2 \mu \mathrm{m}$ buried oxide layer. The PCG design is based on the Rowland geometry with one stigmatic (i.e. aberration-free) point for the wavelength of $2.32 \mu \mathrm{m}$ [3]. The grating diffraction order $m$ is 50, which corresponds to a free spectral range of $34 \mathrm{~nm}$ at $2.32 \mu \mathrm{m}$. The PCG design is suitable for glucose absorption spectroscopy.

The entrance and exit waveguides are positioned along the Rowland circle with a radius of $806 \mu \mathrm{m}$ and the curved grating follows a circle with a radius of $1612 \mu \mathrm{m}$. To optimize the reflection of the grating facets, we use first 
order distributed Bragg reflector (DBR) facets with a period of $540 \mathrm{~nm}$, exhibiting a simulated reflectivity of $>80 \%$ over the 2-2.5 $\mu \mathrm{m}$ range. The centers of the exit waveguide apertures are spaced $7.6 \mu \mathrm{m}$ apart. The entrance and exit apertures are $3 \mu \mathrm{m}$ wide, shallowly etched (70 $\mathrm{nm}$ deep) waveguides. We use a double adiabatic taper to convert from these apertures to the deeply etched, $800 \mathrm{~nm}$ wide, photonic wire routing waveguides. These photonic wires have a measured propagation loss of $2.5 \mathrm{~dB} / \mathrm{cm}$ for the TE mode.

The PCG is characterized using a short-wave-infrared laser, operating in amplified spontaneous emission (ASE) mode, and an optical spectrum analyzer with $0.375 \mathrm{~nm}$ spectral resolution. The ASE light is coupled into a single mode optical fiber and a polarization controller is used to excite the TE mode. The in- and outputs of the PCG are accessed with optical fibers through grating couplers. The measured on-chip transmission response of the PCG and fabricated device are shown in Fig. 1(a) and (b) respectively. The grating couplers are first generation components with a peak efficiency at $2.15 \mu \mathrm{m}$, instead of the design wavelength of $2.32 \mu \mathrm{m}$. Considering this shift in operation wavelength, the central channel insertion loss of $4 \mathrm{~dB}$ is relatively low. This indicates a robust PCG design. The near-channel crosstalk ranges from $-12 \mathrm{~dB}$ to $-10 \mathrm{~dB}$, although the optimum values are probably masked by the high noise floor of the set-up. The $6^{\text {th }}$ channel has a higher insertion loss due to a local design error as indicated by an arrow in Fig. 1(c).

\section{GaSb-on-SOI photodetector}
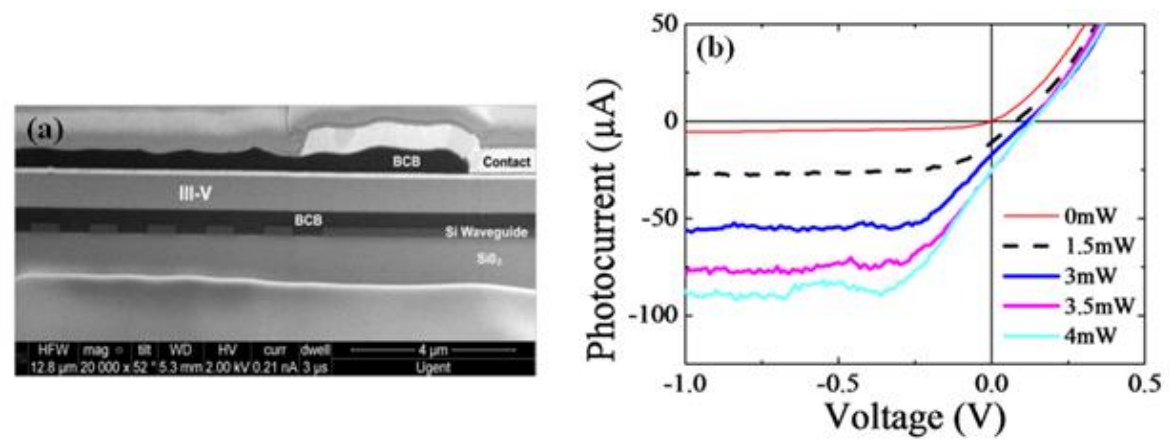

Fig. 2. (a) SEM image of the fabricated photodiode (b) IV-characterization of the photodiode at different optical fiber input power levels

The integration of GaSb-based photodetectors is realized through an epitaxial layer transfer process using BCB adhesive bonding. The p-i-n detector has a $500 \mathrm{~nm}$ thick $\mathrm{Ga}_{0.79} \mathrm{In}_{0.21} \mathrm{As}_{0.19} \mathrm{Sb}_{0.81}$ intrinsic absorption layer and metal contacts composed of $\mathrm{Au}$ with $\mathrm{Ti}(2 \mathrm{~nm}) / \mathrm{Pt}(35 \mathrm{~nm})$. The surface area of the photodetector mesa is $50 \times 20 \mu \mathrm{m}^{2}$. Fig. 2(a) shows a SEM picture of a cross-section of the fabricated grating-assisted photodetector. The fabrication procedure can be found in [4]. To characterize the photodetector, we inject TE-polarized light from a short-wave infrared laser through grating couplers into the waveguide circuit and measure the photoresponse at a wavelength of $2.2 \mu \mathrm{m}$ for different fiber input power levels. The resulting voltage-current curve is plotted in Fig. 2(b). The dark current density at $-1 \mathrm{~V}$ is determined to be $0.4 \mathrm{~A} / \mathrm{cm}^{2}$ at room temperature. In order to decrease the dark current value, we are currently studying several passivation methods. The system sensitivity, from input fiber to detector, is $25 \mathrm{~mA} / \mathrm{W}$. Given the (low) measured grating coupler efficiency of $-13 \mathrm{~dB}$, we can deduce from this sensitivity value that the detector responsivity amounts to $0.5 \mathrm{~A} / \mathrm{W}$.

\section{Conclusion}

We report on the first silicon-based PCG for short-wave infrared applications. The characterization of the first grating-assisted GaSb photodetector shows the diode's good opto-electronic performance. Based on this photodiode design, we can thus proceed with the fabrication of a photodetector array suitable for the integrated spectrometer.

[1] A. Bedoya et al., "Biodiesel sensing using Silicon-on-Insulator technologies," in SPIE Europe, Microtechnologies for the new millennium, 2009

[2] N. Hattasan et al., "Heterogeneous integration of GaInAsSb p-i-n photodiodes on a silicon-on-insulator waveguide circuit," IEEE Photonics Technology Letters 23, 1760-1762 (2011)

[3] R. März, Integrated optics, design and modeling (Artech House Inc. ,1994).

[4] N. Hattasan et al., "Heterogeneously integrated InGaAsSb detectors on SOI waveguide circuits for short-wave infrared applications", in Integrated Photonics Research, Silicon and Nanophotonics, OSA Technical Digest (CD), 2011 